\title{
Consumo de cannabis entre adolescentes: patrón de riesgo, implicaciones y posibles variables explicativas
}

\section{Cannabis use among adolescents: Risk pattern, implications and possible explanatory variables}

\author{
Antonio Rial*, Gregor Burkhart**, Manuel Isorna***, \\ Carmen Barreiro*, Jesús Varela*, Sandra Golpe*. \\ * Universidad de Santiago de Compostela, Santiago de Compostela, España. ** European Monitoring \\ Centre for Drugs and Drug Addiction (EMCDDA). *** Universidad de Vigo, Vigo, España.
}

\section{Resumen}

Contrariamente a lo que ocurre con el resto de sustancias todavía no se han logrado disminuir los niveles de consumo de cannabis, que sigue siendo la droga ilegal más consumida entre los adolescentes españoles. El objetivo de este estudio consiste en actualizar los niveles de consumo (incorporando la franja de edad de 12-13 años), estimar los consumos propiamente de riesgo y analizar las posibles variables asociadas. Para ello se ha utilizado una metodología correlacional consistente en la realización de una encuesta a estudiantes de ESO y Bachillerato de la comunidad autónoma de Galicia (España) en el año 2016. La muestra final estuvo compuesta por 3.882 adolescentes gallegos de entre 12 y 18 años $(M=14,52$ y $D T=1,72)$. Los resultados obtenidos revelan que a día de hoy es ya mayor el porcentaje de adolescentes que consumen tabaco y cannabis que únicamente tabaco (12,7\% vs $10,5 \%)$ y que ello no sólo implica una mayor probabilidad de consumir otras sustancias ilegales, sino también de desarrollar un patrón consumo de alcohol de riesgo, de Binge Drinking o incluso de experimentar un Uso Problemático de Internet o de ciberacoso. Las variables personales como la autoestima, la asertividad, las habilidades sociales o la impulsividad tienen una capacidad explicativa realmente débil, en comparación con otras variables vinculadas al establecimiento de normas y límites por parte de los padres. Una de las principales conclusiones de este trabajo es la necesidad de adoptar un enfoque de prevención integral.

Palabras clave: Adolescentes; Cannabis; Consumo; Tabaco; Variables asociadas.

\begin{abstract}
In contrast to the achievements with other substances, it has not yet been possible to reduce the levels of cannabis use, the most used illegal substance among Spanish adolescents. The objective of this paper consists of updating levels of use (incorporating ages 12 and 13), estimating high-risk use and analyzing possible associated variables. For this purpose, a correlational method was used consisting of the administration of a survey to compulsory secondary school and high school students from the autonomous community of Galicia (Spain) in 2016. Results obtained from a sample of 3,882 Galician adolescents aged 12 to $18(\mathrm{M}=14.52 ; \mathrm{SD}=1.72)$ reveal that the percentage of adolescents currently using tobacco and cannabis is higher than that of those using tobacco alone $(12.7 \%$ vs $10.5 \%)$. This implies not only a higher probability of using other illegal substances, but also of developing rather high-risk use pattern, binge drinking or even experiencing problematic Internet use or cyberbullying. From a preventive perspective, the results reveal that personal variables such as self-esteem, assertiveness, social skills or impulsiveness have really weak explanatory power compared with other variables related to the setting of rules and limits by parents. One of the main conclusions of this paper is the need to adopt a comprehensive prevention approach. Key words: Adolescents; Cannabis; Consumption; Tobacco; Related variables.
\end{abstract}


L os datos de la última Encuesta Estatal sobre el Uso de Drogas en Enseñanzas Secundarias (ESTUDES 2014/15) (Plan Nacional sobre Drogas, 2016) ponen de manifiesto que, a diferencia de lo que ocurre con el resto de las sustancias, las cifras de consumo de cannabis apenas han descendido respecto a la edición anterior, siendo la droga ilegal más consumida entre los adolescentes de entre 14 y 18 años. Más allá de los niveles de consumo el propio ESTUDES 2014/15 revela que un 2,5\% de los adolescentes españoles presenta un patrón de consumo realmente de riesgo, al dar positivo en el Cannabis Abuse Screning Instrument (CAST) (Legleye, Piontek y Kraus, 2011).

Por otro lado, hay trabajos que han advertido de un descenso en las edades de inicio de consumo de las diferentes sustancias, entre ellas el cannabis (Golpe, Barreiro, Isorna, Gómez y Varela, 2016), recalcando las consecuencias que un inicio precoz en el consumo puede llevar asociadas (Brook, Stimmel, Zhang y Brook, 2008; Hérnandez, Roldán, Jiménez, Mora, Escarpa, 2009; Kokkevi, Gabhainn y Spyropoulou, 2006). Los datos disponibles a nivel oficial (Plan Nacional sobre Drogas, 2014) sitúan la edad de inicio del consumo de cannabis en los 14,9 años; dato que posiblemente no representen de forma precisa la realidad, habida cuenta del marco muestral utilizado (14-18 años).

Si bien hay autores que defienden que cada vez un mayor número de adolescentes se inician ya antes en el consumo de cannabis que en el de tabaco (Álvarez et al., 2016; Rolle et al., 2015), las teorías clásicas postulan que el consumo de alcohol y tabaco antecede al consumo de cannabis y éste, a su vez, al de otras drogas ilícitas (Kandel, 2003). En esa línea autores como Morral, McCaffrey y Paddock (2002) o Swift et al. (2011) sostienen que dar el paso al consumo de cannabis supone un salto cualitativo que incrementaría el riesgo de "progresar" hacia el consumo de otras drogas ilegales, lo cual lo convierte en una cuestión de enorme trascendencia. No hay que olvidar tampoco que cuando los adolescentes se incorporan al consumo de cannabis, por lo general siguen manteniendo su consumo de tabaco (Álvarez et al., 2016; Ariza et al., 2014). Tanto es así que la forma más común de consumo de esta sustancia entre la mayoría de los consumidores europeos es mezclada con éste (Font-Mayolas et al., 2013; Pirona, Noor y Burkhart, 2015). Su consumo combinado no solo incide en el mantenimiento del consumo de ambas sustancias, sino que incrementa también su potencial adictivo (Hindocha et al., 2016; Tullis, Dupont, Frost y Gold, 2003), con implicaciones severas a nivel neurobiológico cuyo estudio debiera acaparar, según Pirona et al. (2015), mayor atención de la que ha merecido hasta hoy.

Además de lo señalado existen una serie de elementos sociosanitarios que justifican la necesidad de seguir avanzando en el estudio del cannabis (Isorna, 2017). Numerosos trabajos han hecho hincapié en las implicaciones que el consumo de dicha sustancia tiene a nivel cerebral, tanto desde un punto de vista estructural como funcional (Jacobus y Tapert, 2014). Se han constatado además efectos adversos para la salud a nivel respiratorio y cardiovascular (Bechtold, Simpson, White y Pardini, 2015). Se ha asociado a una mayor prevalencia de sintomatología psicótica (Arseneault et al., 2002; Henquet et al., 2005), ansiedad, depresión o un mayor riesgo de suicidio (Feingold, Weiser, Rehm y Lev-Ran, 2016; Silins et al., 2014). Desde un punto de vista psicosocial se ha constatado que los adolescentes que consumen cannabis presentan mayores dificultades para estudiar o trabajar, tienen un peor rendimiento académico y se implican en más conflictos o discusiones, así como en peleas o agresiones físicas (Guerrero et al., 2015; Morales, Ariza, Nebot, Pérez y Sáchez, 2008; Plan Nacional sobre Drogas, 2014) y prácticas sexuales de riesgo (Harper, Dittus y Ethier, 2016). Por otro lado, el consumo de esta sustancia en la adolescencia ha sido relacionado con una mayor probabilidad de desarrollar una posible dependencia en la edad adulta (George y Vaccarino, 2015) y con el consumo de otras drogas (Fergusson y Boden, 2008; Hurd, Michaelides, Miller y Jutras-Aswad, 2014). Se ha evidenciado también que conducir bajo los efectos de esta droga incrementa el riesgo de sufrir accidentes de tráfico (Asbridge, Hayden y Cartwright, 2012; Gerberich et al., 2003; Hartman y Huestis, 2013). Por último, no menos importantes son los estudios que han relacionado el uso de esta sustancia con el Uso Problemático de Internet (Golpe, Gómez, Braña, Varela y Rial, 2017; Rücker, Akré, Berchtold y Suris, 2015) y diferentes prácticas de riesgo on line, tales como el sexting (Benotsch, Snipes, Martin y Bull, 2013), el cyberbullying (Halbohn, 2016) o el uso problemático de los videojuegos online (Van Rooij et al., 2014).

Las implicaciones que el consumo de esta sustancia puede acarrear a distintos niveles justifica la necesidad de desarrollar líneas de actuación específicas a nivel de prevención debidamente avaladas por la evidencia científica. Eso es precisamente lo que ha llevado a diferentes investigadores a intentar identificar posibles variables asociadas y tratar de determinar el peso relativo de cada una de ellas como factores de pronóstico. Algunas de las más estudiadas han sido aquellas que tienen que ver con características de personalidad, sobre todo la impulsividad (Barkus, 2008; Moreno et al., 2012) y la búsqueda de sensaciones (González, Sáiz, Quirós y López, 2000; Malmberg et al., 2010). Otros trabajos se han centrado en analizar el papel de las habilidades sociales (Griffith-Lendering et al., 2011). Sin embargo, en los últimos años han ido adquiriendo cada vez mayor importancia los modelos basados en el enfoque de la prevención ambiental (Burkhart, 2011), desde los que se asume que las personas no se involucran en el consumo de sustancias solo por razones personales o cognitivas, sino que se ven influenciados por factores ambientales. Trabajos como los de Guxens, Nebot, Ariza y Ochoa (2007) o el 
de Thompson y Auslander (2007) recalcan la importancia del consumo de cannabis en el círculo de amistades del adolescente. Terzic, Santric, Sbutega y Vasic (2013), por su parte, advierten que un contexto familiar desestructurado o una mala relación con los padres se asocian también al consumo de esta sustancia, mientras que Vázquez et al. (2014) hace énfasis en la hora de llegada a casa y Varela, Marsillas, Isorna y Rial (2013) en el dinero disponible.

Habida cuenta de todas estas consideraciones de partida, el presente trabajo se plantea con un triple objetivo: (1) por un lado actualizar la prevalencia de consumo tanto de cannabis como de otras sustancias psicoactivas entre los adolescentes, así como las edades de inicio y los consumos propiamente de riesgo, incorporando al marco muestral objeto de estudio a los adolescentes de 12 y 13 años; (2) a pesar de que no es posible establecer relaciones causa efecto, el segundo objetivo de este trabajo consiste en analizar la relación entre el policonsumo de tabaco y cannabis y otras conductas, tratando de explorar posibles implicaciones como el consumo de otras sustancias, el consumo de riesgo de alcohol y binge drinking, el Uso Problemático de Internet o determinadas prácticas de riesgo en la Red; y (3) analizar el papel de posibles factores de pronóstico tanto de índole personal (como la autoestima, la impulsividad, las habilidades sociales o la asertividad), como familiar (fundamentalmente el dinero disponible y la hora de llegada a casa).

\section{Método}

\section{Participantes}

Para dar cuenta de los objetivos señalados se recurrió a una metodología selectiva, consistente en la realización de una encuesta entre estudiantes de Educación Secundaria Obligatoria (ESO) y Bachillerato de las provincias de A Coruña y Pontevedra. En concreto se ha aplicado un diseño transversal correlacional. Para la selección de la muestra se utilizó un muestreo bietápico, por conglomerados para la selección de las unidades de primer nivel (centros) -estableciendo cuotas a priori en función de la titularidad de éstos-y un muestreo intencionado para la selección de unidades de segundo nivel (individuos). A pesar del carácter no probabilístico del muestreo utilizado, se comprobó que las cuotas muestrales finales se ajustasen a las cuotas poblacionales, en lo que se refieren al sexo, la edad, el curso académico y la titularidad del centro educativo. Aceptaron participar en el estudio 15 centros educativos de diferentes municipios, tanto públicos como privados y concertados, de carácter tanto urbano como rural. El número inicial de cuestionarios recogidos fue de 4063, si bien fueron eliminados 62 tras el proceso de revisión, bien por presentar un excesivo número de valores missing $(\mathrm{n}=32)$ o patrones de respuesta incoherentes $(n=30)$. Posteriormente se eliminaron otros 119 casos por encontrarse fuera del rango de edad objeto de estudio (12-18 años). Como consecuencia la muestra final estuvo compuesta de 3882 adolescentes (49,9\% hombres y $50,1 \%$ mujeres) de edades comprendidas entre los 12 y 18 años $(M=14,52$ y $D T=1,72)$. De estos, 2669 asistían a colegios públicos y 1213 a colegios privados o concertados. El 74,8\% se encontraban cursando ESO (38\% en el primer ciclo y $36,8 \%$ en el segundo) y el 25,2\% Bachillerato.

\section{Instrumento}

Los datos fueron recogidos mediante un cuestionario elaborado expresamente para el presente estudio, en el que se incluían preguntas agrupadas en cinco bloques. Un primer bloque extraído del ESTUDES 2014/15 (Plan Nacional sobre Drogas, 2016) que contenía preguntas referidas a los hábitos de consumo tanto de alcohol, borracheras y binge drinking (definido como "la ingesta de 6 o más bebidas alcohólicas por ocasión de consumo", de acuerdo con trabajos como los de Parada et al., 2011 o Golpe et al., 2017), consumo de tabaco, cannabis, cocaína, éxtasis, anfetaminas y alucinógenos, tanto en el último año como en el último mes. Un segundo bloque que incluía tres herramientas específicas de screening. Por un lado el Cannabis Abuse Screening Test (CAST, Legleye et al., 2011), que permite identificar a los adolescentes que están realizando un consumo de riesgo de cannabis. A pesar de que el CAST permite establecer tres categorías en función de la puntuación total obtenida ("ningún riesgo" = 0-1 puntos; "bajo riesgo" = 2-3 puntos; "alto riesgo" = 4 o más puntos), siguiendo el propio ESTUDES 2014-2015, se ha utilizado únicamente el punto de corte 4 , distinguiendo entre los adolescentes que están realizando un consumo abusivo o problemático de cannabis de aquellos que no consumen o cuyo consumo no los sitúa todavía en una situación de alto riesgo. Por otra parte, para identificar a los consumidores de riesgo de alcohol se utilizó la versión del Alcohol Use Disorders Identification Test (AUDIT), recientemente validada con adolescentes españoles por Rial, Golpe, Araujo, Braña y Varela (2017), que utiliza también como punto de corte una puntuación $\geq$ a 4 . Por último, para identificar a los usuarios problemáticos de Internet, se aplicó la Escala de Uso Problemático de Internet para adolescentes (EUPI-a, de Rial, Gómez, Isorna, Araujo y Varela, 2015), con una puntuación de corte $\geq$ a 16 , tal y como recomiendan los autores. En un tercer bloque se pretendía evaluar diferentes conductas de riesgo en Internet, como el sexting, la participación en juegos de azar on line y apuestas en la Red, cyberbullying, contacto con desconocidos, etc. El cuarto bloque incluía preguntas referidas a posibles variables asociadas al consumo de cannabis. Entre ellas se exploraron variables personales como la autoestima (a través de la Escala de Autoestima de Rosenberg, adaptada por Martín, Núñez, Navarro y Grijalvo, 2007); la asertividad (a través de la Escala Asertividad incluida en el Banco de Instrumentos para la 
Tabla 1. Consumo de las diversas sustancias por los adolescentes escolarizados por sexo y grupos de edad, según sustancia. Galicia Occidental, 2016.

\begin{tabular}{|c|c|c|c|c|c|c|c|c|c|}
\hline \multirow[t]{2}{*}{ ÚLTIMO AÑO } & \multicolumn{2}{|c|}{ GLOBAL } & \multicolumn{2}{|c|}{ SEXO } & \multicolumn{5}{|c|}{ GRUPO EDAD } \\
\hline & $\begin{array}{c}12-18 \text { años } \\
\%(n)\end{array}$ & $\begin{array}{c}\text { 14-18 años } \\
\%(n)\end{array}$ & $\begin{array}{l}\text { Hombre } \\
\%(n)\end{array}$ & $\begin{array}{l}\text { Mujer } \\
\%(n)\end{array}$ & $p^{\star}$ & $\begin{array}{c}\text { 12-13 años } \\
\%(n)\end{array}$ & $\begin{array}{c}\text { 14-15 años } \\
\%(n)\end{array}$ & $\begin{array}{c}16-18 \text { años } \\
\%(n)\end{array}$ & $p^{\star}$ \\
\hline Cannabis & $14,8(575)$ & $19,8(509)$ & $15,3(295)$ & $14,3(277)$ & ,42 & $4,5(57)$ & $12,6(171)$ & 27,9 (338) & $<, 001$ \\
\hline Alcohol & $52,1(2021)$ & $64,3(1655)$ & $50,7(980)$ & $53,4(1035)$ &, 10 & $27(344)$ & $54,5(741)$ & 75,4 (914) & $<, 001$ \\
\hline Emborracharse & $26,3(1017)$ & $34,4(882)$ & $25,4(490)$ & $27(522)$ &, 28 & $9,4(120)$ & 21,6 (293) & 48,6 (589) & $<, 001$ \\
\hline Tabaco & $23,4(905)$ & $30(768)$ & $21,2(410)$ & $25,4(491)$ &, 002 & $9,7(124)$ & $21,3(289)$ & $39,6(479)$ & $<, 001$ \\
\hline Cocaína & $0,9(34)$ & $1(26)$ & $1,1(21)$ & $0,6(12)$ & ,16 & $0,5(7)$ & $1(13)$ & $1,1(13)$ & ,32 \\
\hline $\begin{array}{l}\text { Éxtasis/ } \\
\text { anfetaminas/ } \\
\text { alucinógenos }\end{array}$ & $1,1(42)$ & $1,4(35)$ & $1,2(24)$ & $0,9(17)$ &, 34 & $0,5(6)$ & $0,9(12)$ & $1,9(23)$ &, 002 \\
\hline \multirow[t]{2}{*}{ ÚLTIMO MES } & \multicolumn{2}{|c|}{ GLOBAL } & \multicolumn{2}{|c|}{ SEXO } & \multicolumn{5}{|c|}{ GRUPO EDAD } \\
\hline & $\begin{array}{c}12-18 \text { años } \\
\%(n)\end{array}$ & $\begin{array}{c}\text { 14-18 años } \\
\%(n)\end{array}$ & $\begin{array}{l}\text { Hombre } \\
\%(n)\end{array}$ & $\begin{array}{l}\text { Mujer } \\
\%(n)\end{array}$ & $p^{\star}$ & $\begin{array}{c}12-13 \text { años } \\
\%(n)\end{array}$ & $\begin{array}{c}\text { 14-15 años } \\
\%(n)\end{array}$ & $\begin{array}{c}\text { 16-18 años } \\
\%(n)\end{array}$ & $p^{\star}$ \\
\hline Cannabis & $8,5(330)$ & $11,4(294)$ & $8,6(166)$ & $8,4(162)$ & ,84 & $2,6(33)$ & $6,6(90)$ & $16,8(204)$ & $<, 001$ \\
\hline Alcohol & $32,3(1253)$ & 41,3 (1062) & $30(580)$ & $34,5(669)$ &, 003 & $13,6(174)$ & 29,1 (394) & $55,1(668)$ & $<, 001$ \\
\hline Emborracharse & $12,9(499)$ & $17,2(442)$ & $11,7(225)$ & $14(272)$ &, 03 & $3,8(49)$ & $9,3(126)$ & $26,1(316)$ & $<, 001$ \\
\hline $\begin{array}{l}6 \text { o más bebidas } \\
\text { alcohólicas }\end{array}$ & $8,6(334)$ & $11,4(293)$ & 8,9 (171) & $8,3(161)$ & 0,57 & $2,6(33)$ & $6,6(89)$ & $16,8(204)$ & $<, 001$ \\
\hline Tabaco & $16,1(623)$ & 20,7 (532) & $14,3(276)$ & $17,8(345)$ &, 003 & $6,7(85)$ & 14,1 (191) & $28,2(341)$ & $<, 001$ \\
\hline Cocaína & 0,4 (15) & $0,3(8)$ & $0,4(7)$ & $0,4(7)$ & 1 & $0,5(6)$ & $0,4(6)$ & $0,2(2)$ & ,38 \\
\hline $\begin{array}{l}\text { Éxtasis/ } \\
\text { anfetaminas/ } \\
\text { alucinógenos }\end{array}$ & 0,4 (16) & $0,4(11)$ & $0,3(6)$ & $0,5(9)$ & ,61 & $0,3(4)$ & $0,5(7)$ & $0,3(4)$ & 65 \\
\hline
\end{tabular}

Nota. * Significación asociada al contraste Chi-cuadrado.

Evaluación de Intervenciones Preventivas del Observatorio Europeo de las Drogas y las Toxicomanías [OEDT]; la impulsividad (mediante la Escala de Impulsividad de Barrat, adaptada por Martínez, Fernández, Fernández, Carballo y García, 2015); y las habilidades sociales (a través de la Escala de Habilidades Sociales de Oliva et al., 2011). Para conocer la hora de llegada a casa y el dinero disponible se utilizaron dos preguntas extraídas del trabajo de Varela et al. (2013). Por último, en un quinto bloque se recogieron datos de carácter sociodemográfico como sexo, edad y titularidad del centro.

\section{Procedimiento}

Los datos fueron recogidos en las propias aulas de los centros, en grupos reducidos (entre 15 y 20 individuos), mediante un cuestionario que cada adolescente debía cumplimentar de manera individual. La recogida de la información tuvo lugar en el primer trimestre de 2016 y fue realizada por un equipo de psicólogo/as con experiencia acreditada en la realización de este tipo de tareas. Cada sujeto fue informado de la finalidad del estudio, garantizando la confidencialidad y el anonimato de sus respuestas.
Se contó con el consentimiento y la colaboración tanto de la dirección de los centros, como de las respectivas asociaciones de madres y padres de alumnos. La participación fue totalmente voluntaria y el tiempo de cumplimentación del cuestionario fue de aproximadamente 20 minutos. El trabajo contó además con la aprobación del Comité de Bioética de la Universidad de Santiago de Compostela.

\section{Análisis de datos}

Tras un primer análisis descriptivo y una tabulación bivariada se establecieron tres grupos de comparación. El primero de ellos, formado por aquellos adolescentes que no consumieron ni tabaco ni cannabis en el último año, el segundo, formado por aquellos que solo consumieron tabaco y el tercero, formado por quienes consumieron ambas sustancias. El 2,1\% de adolescentes que solo consumía cannabis fue excluido del análisis. Las diferencias entre los grupos fueron analizadas mediante la aplicación de contrastes paramétricos y no paramétricos, en función de la naturaleza de las variables. En el caso de variables cuantitativas se realizaron Anova unifactoriales con contrastes post-hoc de 
Tukey y el coeficiente eta cuadrado parcial $\left(\eta_{p}^{2}\right)$ para estimar el tamaño del efecto. En el caso de variables cualitativas se aplicaron contrastes de independencia $\chi^{2}$ y coeficientes de contingencia (CC). Los análisis fueron realizados mediante el paquete estadístico IBM SPSS Statistics 20.

\section{Resultados}

\section{Niveles de consumo y edades de inicio}

Tal y como se puede observar en la Tabla 1, el alcohol sería la sustancia más consumida por los adolescentes de entre 12 y 18 años, seguida del tabaco y el cannabis. En concreto, más de la mitad de los adolescentes de la mues- tra $(52,1 \%)$ bebieron alcohol en el último año, 1 de cada 4 se emborrachó y el 18,1\% consumió 6 o más bebidas alcohólicas en una misma ocasión o episodio de consumo. El 23,4\% fumó tabaco y el 14,8\% consumió cannabis. Si se analizan los niveles de consumo con la muestra reducida de adolescentes de entre 14 y 18 años se observa un ligero incremento para todas las sustancias. En lo que respecta a la franja de edad más precoz y que hasta el momento no ha sido incorporada al ESTUDES (12-13 años), los resultados revelan que un $27 \%$ bebió alcohol en el último año (13,6\% en el último mes), el 9,4\% consumió tabaco $(6,7 \%$ en el último mes) y el 4,5\% cannabis (2,6\% en el último mes). Si se analizan los resultados en función del sexo apenas se

Tabla 2. Consumo de diversas sustancias y consumo de riesgo de alcohol por adolescentes escolarizados por sexo y grupo de edad. Galicia Occidental, 2016.

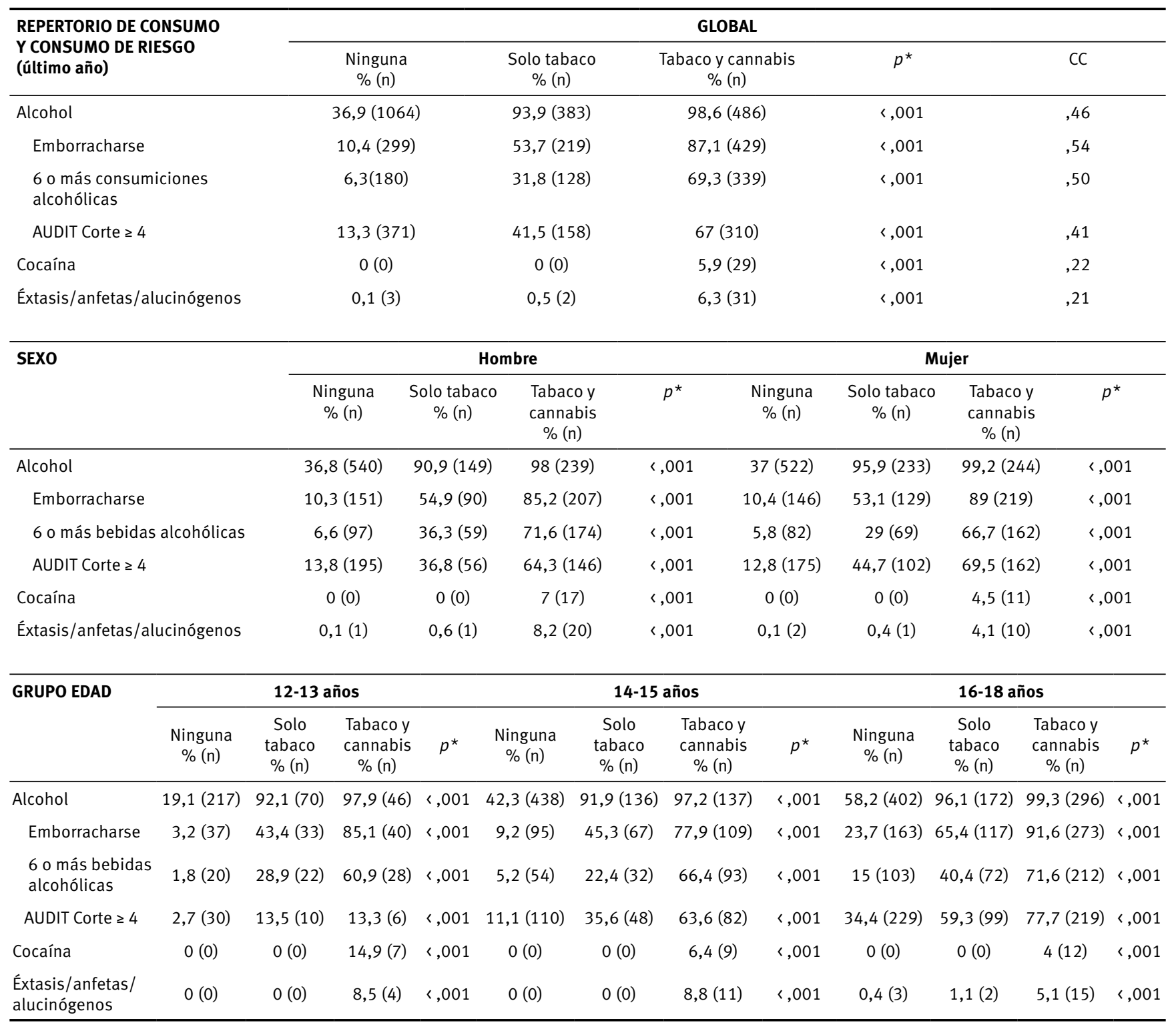

Nota. * Significación asociada al contraste Chi-cuadrado. 
han encontrado diferencias entre chicos y chicas en el consumo de las distintas sustancias y cuando tales diferencias existen (consumo de tabaco en el último año y alcohol, borracheras y tabaco en el último mes), las cifras de prevalencia son mayores entre las chicas. Atendiendo al grupo de edad las diferencias se hacen más que evidentes, siendo el porcentaje de consumidores significativamente mayor entre los adolescentes de 16-18 años.

Por otra parte, las edades de inicio del consumo de tabaco y de cannabis se sitúan en los 14,08 y 14,8 respectivamente, siendo la edad de inicio del consumo de otras sustancias como el alcohol de 13,6 años, la de la primera borrachera de 14,6, la de la cocaína 15,08 y la del consumo de éxtasis, anfetaminas o alucinógenos de 14,9. Cabe añadir además que el $56,3 \%$ de los que probaron el tabaco alguna vez en su vida lo hicieron con 14 años o menos, siendo este porcentaje del $38,8 \%$ en el caso del cannabis.

\section{Consumo de riesgo}

Los datos recogidos en la Tabla 1 revelan que un 3,8\% de los adolescentes de la muestra supera el punto de corte establecido por los autores originales del CAST $(\geq 4)$ o, lo

Tabla 3. Consumo de tabaco y cánnabis según prácticas de riesgo en la red y uso problemático de Internet en adolescentes escolarizados por sexo y grupo de edad. Galicia Occidental, 2016.

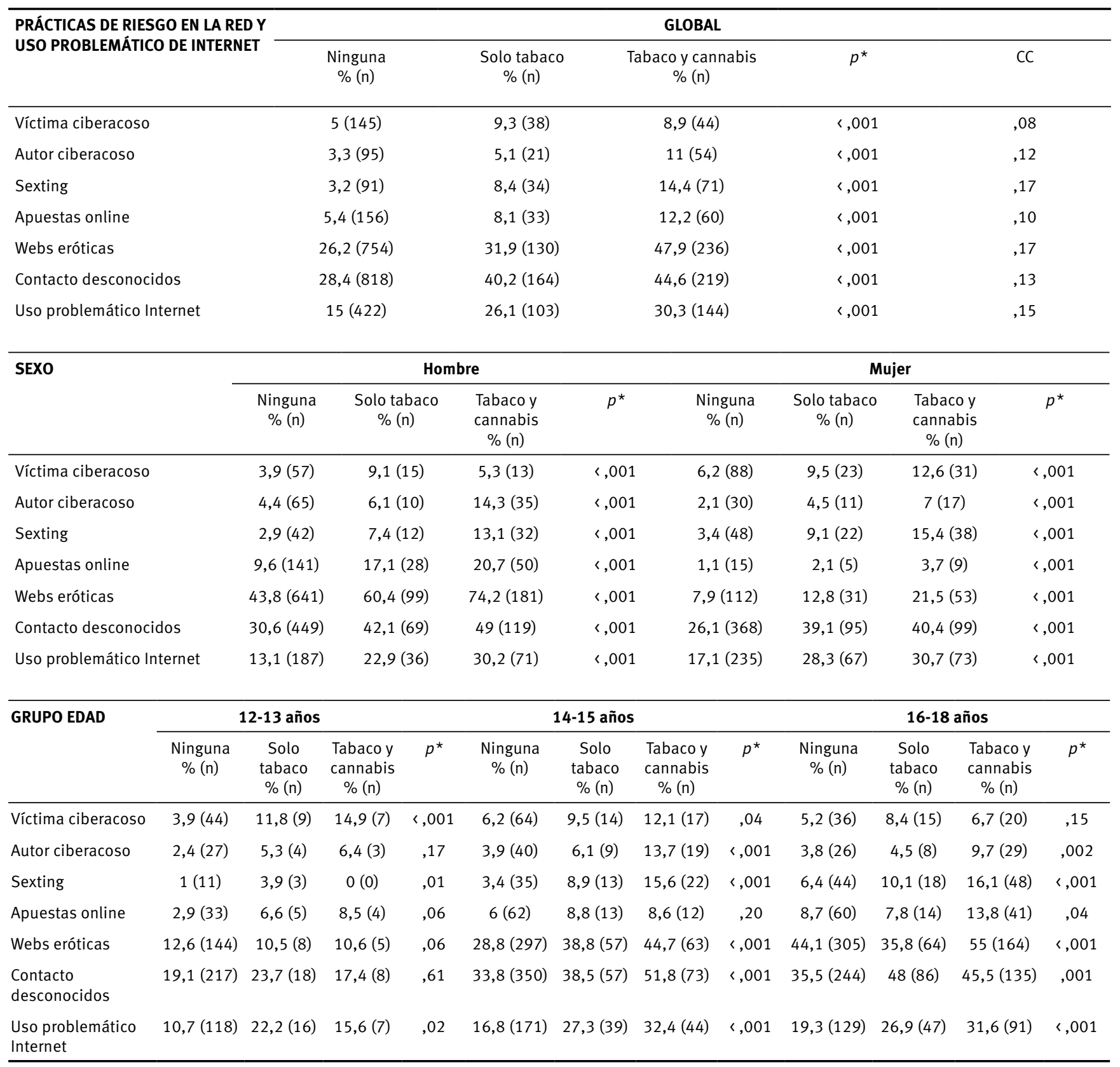

Nota. * Significación asociada al contraste Chi-cuadrado. 


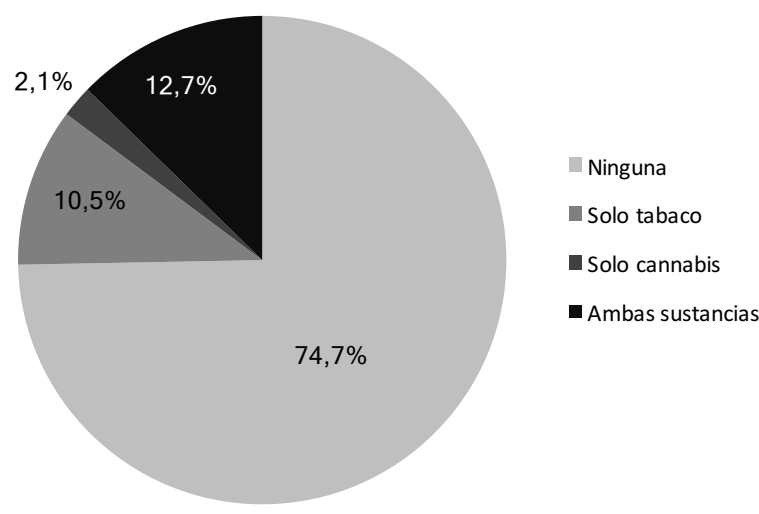

Figura 1. Consumo de tabaco, cannabis y consumo combinado por adolescentes escolarizados. Galicia Occidental, 2016.

que es lo mismo, uno de cada cuatro $(25,4 \%)$ de los que consumieron cannabis en el último año y algo más de uno de cada tres $(37,5 \%)$ de quienes lo consumieron en el último mes presentaría un consumo de alto riesgo de dicha sustancia. A este porcentaje habría que añadir un $2,1 \%$ de adolescentes que presentaría un consumo de bajo riesgo (de 2 a 3 puntos en el CAST). Frente a éstos cabe referirse a un $94,1 \%$ que no presentaría ningún riesgo.

No se han encontrado diferencias significativas atendiendo al sexo, pero sí en función de la edad, encontrando una tasa de consumidores de riesgo 5 veces mayor entre los adolescentes de 16-18 años (6,3\%) que entre los de 12-13 $(1,3 \%)\left(\chi^{2}=44,52 ; p<, 001\right)$.

\section{Relación entre el consumo de tabaco y de cannabis}

Si se analiza qué ocurre de manera específica con el caso del tabaco y el cannabis, un primer dato de interés es que la mayoría de los adolescentes $(74,7 \%)$ no consume ninguna de las dos sustancias. Por otra parte, se ha encontrado que hay más adolescentes que fuman ambas sustancias $(12,7 \%)$ que sólo tabaco $(10,5 \%)$, tratándose de una diferencia estadísticamente significativa $\left(\chi^{2}=8,02 ; p<, 01\right)$. El porcentaje de adolescentes que consume únicamente cannabis ha resultado ser muy bajo $(2,1 \%)$. También se ha podido constatar que el $54,7 \%$ de los que fumaron tabaco en el último año también consumieron cannabis, mientras que el $86 \%$ de los que consumieron cannabis también fumaron tabaco (Figura 1).

\section{Otras prácticas de riesgo}

Tal y como se puede observar en la Tabla 2, la tasa de consumidores de otras sustancias es significativamente mayor entre aquellos adolescentes que consumen tabaco y cannabis en comparación con los otros dos grupos, especialmente en lo que se refiere al consumo intensivo de alcohol. Así, por ejemplo, se observa que el porcentaje de adolescentes que reconocieron haberse emborrachado en el último año pasa del 10,4\% entre los que no consumie- ron ninguna de las dos sustancias, al $87,1 \%$ entre aquellos que consumieron tabaco y cannabis $\left(\chi^{2}=1591,02 ; p<, 001\right.$; $\mathrm{CC}=, 54)$. Lo mismo ocurre para la ingesta de 6 o más bebidas alcohólicas por ocasión de consumo, que pasa del $6,3 \%$ al $69,3 \%\left(\chi^{2}=1269,17 ; p<, 001 ; \mathrm{CC}=, 50\right)$. Asimismo, las tasas de consumo de cocaína, éxtasis, anfetaminas y alucinógenos también son significativamente mayores aunque presentan tamaños de efecto más moderados. Por otra parte, se ha podido constatar que la incorporación del cannabis al repertorio de consumo se asocia también a un mayor consumo de riesgo. Tanto es así que la tasa de consumidores de riesgo de alcohol, evaluada a través del AUDIT, es 5 veces mayor en el caso de los adolescentes que fuman tabaco y cannabis $(13,3 \%$ vs $67 \%)\left(\chi^{2}=764,26 ; p<\right.$ $, 001 ; \mathrm{CC}=, 41)$. Los análisis realizados por sexo y por grupo de edad revelan que estas diferencias se mantienen tanto para el caso de los chicos como de las chicas y para todos los grupos de edad.

En lo que se refiere al comportamiento de los adolescentes en la Red (Tabla 3) se ha encontrado que aquellos que consumen tabaco y cannabis tendrían una probabilidad significativamente mayor de implicarse en distintas conductas de riesgo. En concreto, la magnitud del tamaño del efecto revela que el sexting $\left(\chi^{2}=117,61 ; p<, 001 ; \mathrm{CC}=, 17\right)$ y el acceso a webs de contenido erótico $\left(\chi^{2}=112,61 ; p<, 001\right.$; $\mathrm{CC}=, 17)$ son las prácticas de riesgo que tienen una asociación más estrecha con ese patrón de consumo simultáneo. Asimismo, se ha observado que el porcentaje de usuarios problemáticos de Internet pasa del $26,1 \%$ entre aquellos que consumen tabaco al 30,3\% entre los que consumen tabaco y cannabis, siendo esta cifra el doble de la que se registra con respecto a los que no consumen ninguna sustancia $(15 \%)\left(\chi^{2}=84,34 ; p<, 001 ; \mathrm{CC}=, 15\right)$. Las diferencias halladas entre los distintos grupos de comparación se mantienen independientemente del sexo observando los mayores porcentajes entre los consumidores de ambas sustancias, excepto en el caso de las víctimas de ciberacoso. Mientras que los chicos consumidores de tabaco son los que registran las mayores cifras de prevalencia $(9,1 \%) \quad\left(\chi^{2}=10,75\right.$; $p<, 001)$, en el caso de las chicas los mayores porcentajes se dan entre las que consumen ambas sustancias $(12,6 \%)$ $\left(\chi^{2}=16,49 ; p<, 001\right)$. En función del grupo de edad, cabe referirse a la ausencia de diferencias estadísticamente significativas en la mayoría de las prácticas de riesgo entre los adolescentes de 12-13 años, excepto en el hecho de ser víctima de ciberacoso (conducta de riesgo predominante entre los consumidores de ambas sustancias), el sexting y el Uso problemático de Internet (predominantes entre los consumidores de tabaco). A partir de los 14 años la tendencia observada es la misma que la registrada a nivel global.

\section{Variables asociadas}

En primer lugar, en lo que se refiere a las variables personales (Tabla 4) cabe señalar que si bien se han encontra- 
Tabla 4. Consumo de tabaco y cánnabis y puntuaciones medias en variables personales en adolescentes escolarizados por sexo y grupo de edad. Galicia Occidental, 2016.

\begin{tabular}{|c|c|c|c|c|c|c|c|c|c|c|c|c|}
\hline & \multicolumn{12}{|c|}{ GLOBAL } \\
\hline & \multicolumn{3}{|c|}{$\begin{array}{l}\text { Ninguna } \\
(\mathrm{M})\end{array}$} & \multicolumn{2}{|c|}{$\begin{array}{l}\text { Solo tabaco } \\
\text { (M) }\end{array}$} & \multicolumn{3}{|c|}{$\begin{array}{c}\text { Tabaco y cannabis } \\
\text { (M) }\end{array}$} & \multicolumn{2}{|l|}{$p^{*}$} & \multicolumn{2}{|c|}{$\eta^{2} p$} \\
\hline Autoestima & \multicolumn{3}{|c|}{31,26} & \multicolumn{2}{|c|}{29,47} & \multicolumn{3}{|c|}{30,46} & \multicolumn{2}{|l|}{$<, 001$} & \multicolumn{2}{|l|}{, 06} \\
\hline Impulsividad & \multicolumn{3}{|c|}{62,63} & \multicolumn{2}{|c|}{65,95} & \multicolumn{3}{|c|}{67,78} & \multicolumn{2}{|l|}{$<, 001$} & \multicolumn{2}{|l|}{, 05} \\
\hline Habilidades sociales & \multicolumn{3}{|c|}{53,14} & \multicolumn{2}{|c|}{52,98} & \multicolumn{3}{|c|}{54,61} & \multicolumn{2}{|l|}{, 04} & \multicolumn{2}{|l|}{, 05} \\
\hline Asertividad & \multicolumn{3}{|c|}{19,50} & \multicolumn{2}{|c|}{18,88} & \multicolumn{3}{|c|}{18,64} & \multicolumn{2}{|l|}{$<, 001$} & \multicolumn{2}{|l|}{, 03} \\
\hline \multirow[t]{2}{*}{ SEXO } & \multicolumn{6}{|c|}{ Hombre } & \multicolumn{6}{|c|}{ Mujer } \\
\hline & \multicolumn{2}{|c|}{$\begin{array}{l}\text { Ninguna } \\
(M)\end{array}$} & $\begin{array}{l}\text { Solo tabaco } \\
\text { (M) }\end{array}$ & \multicolumn{2}{|c|}{$\begin{array}{c}\text { Tabaco y } \\
\text { cannabis } \\
\text { (M) }\end{array}$} & $p^{*}$ & \multicolumn{2}{|c|}{$\begin{array}{l}\text { Ninguna } \\
(\mathrm{M})\end{array}$} & $\begin{array}{l}\text { Solo tabaco } \\
\text { (M) }\end{array}$ & \multicolumn{2}{|c|}{$\begin{array}{c}\text { Tabaco y } \\
\text { cannabis } \\
\text { (M) }\end{array}$} & $p^{*}$ \\
\hline Autoestima & 32,14 & & 30,33 & 31 & & $<, 001$ & 30,3 & & 28,88 & 29 , & &, 004 \\
\hline Impulsividad & 63,09 & & 66,51 & 67 & & $<, 001$ & 62,1 & & 65,55 & 68 , & & $<, 001$ \\
\hline Habilidades sociales & 53,01 & & 53,05 & 54 & &, 15 & 53,2 & & 52,99 & 54 & &, 24 \\
\hline Asertividad & 18,65 & & 17,49 & 18 & & $<, 001$ & 20,3 & & 19,84 & 20 , & &, 02 \\
\hline GRUPO EDAD & & $12-13$ & 3 años & & & $14-1$ & 5 años & & & $16-1$ & años & \\
\hline & $\begin{array}{l}\text { Ninguna } \\
\text { (M) }\end{array}$ & $\begin{array}{l}\text { Solo } \\
\text { tabaco } \\
\text { (M) }\end{array}$ & $\begin{array}{c}\text { Tabaco y } \\
\text { cannabis } \\
\text { (M) }\end{array}$ & $p^{\star}$ & $\begin{array}{l}\text { Ninguna } \\
\text { (M) }\end{array}$ & $\begin{array}{c}\text { Solo } \\
\text { tabaco } \\
\text { (M) }\end{array}$ & $\begin{array}{c}\text { Tabaco y } \\
\text { cannabis } \\
\text { (M) }\end{array}$ & $p^{*}$ & $\begin{array}{l}\text { Ninguna } \\
\text { (M) }\end{array}$ & $\begin{array}{c}\text { Solo } \\
\text { tabaco } \\
\text { (M) }\end{array}$ & $\begin{array}{c}\text { Tabaco y } \\
\text { cannabis } \\
\text { (M) }\end{array}$ & $p^{*}$ \\
\hline Autoestima & 31,60 & 30,90 & 30,82 &, 58 & 31,10 & 28,58 & 29,96 & $<, 001$ & 30,94 & 29,56 & 30,68 &, 002 \\
\hline Impulsividad & 61,96 & 64,91 & 62,38 &, 003 & 62,82 & 67,98 & 69,29 & $<, 001$ & 63,27 & 64,60 & 67,81 & $<, 001$ \\
\hline Habilidades sociales & 53,33 & 52,88 & 53,79 &, 78 & 52,88 & 52,19 & 53,10 & ,88 & 53,07 & 53,66 & 55,97 & ,001 \\
\hline Asertividad & 19,50 & 18,26 & 19,16 & 01 & 19,32 & 18,42 & 18,24 & $<, 001$ & 19,74 & 19,53 & 19,10 & ,05 \\
\hline
\end{tabular}

Nota. * Significación asociada al contraste F.

do diferencias estadísticamente significativas en todas ellas (autoestima, impulsividad, asertividad y habilidades sociales), los reducidos tamaños de efecto estimados ponen de manifiesto que no son variables que puedan realmente explicar por sí solas el consumo de cannabis. De hecho solo la impulsividad y la asertividad presentan un patrón claro, siendo los consumidores de tabaco y cannabis los que muestran las mayores puntuaciones en impulsividad y las menores puntuaciones en asertividad. El análisis de los resultados atendiendo al sexo revela diferencias estadísticamente significativas en tres de las cuatro variables personales estudiadas (autoestima, impulsividad y asertividad), aunque la única que sigue presentando un patrón claro es la impulsividad. Tanto los chicos como las chicas consumidores de tabaco y cannabis son los que muestra las mayores puntuaciones en impulsividad $\left(M_{\text {chicos }}=67,21 ; F=\right.$ 14,$83 ; p<, 001$ vs $\left.M_{\text {chicas }}=68,31 ; F=23,05 ; p<, 001\right)$. Atendiendo al grupo de edad, la impulsividad vuelve a ser la variable más consistente, si bien en el grupo de 12 a 13 son los consumidores de tabaco los que presentan unas mayores puntuaciones en esta variable, mientras que a partir de los 14 años las mayores puntuaciones en impulsividad se corresponden con los consumidores de tabaco y cannabis.
Además de estas variables se analizaron otras relacionadas con el establecimiento de normas y límites en el ámbito familiar. En lo que respecta a la hora de llegada a casa, los resultados recogidos en la Tabla 5 revelan que la probabilidad de pertenecer al grupo de riesgo se incrementa significativamente cuanto más tarden lleguen los adolescentes a casa, especialmente a partir de las 4 de la madrugada $\left(\chi^{2}=529,47 ; p<, 001\right)$. Esta misma tendencia se mantiene cuando se analizan los resultados tanto por sexo como por grupo de edad. Por último, también se ha podido constatar diferencias estadísticamente significativas en función del dinero disponible (Tabla 6). De este modo, la probabilidad de consumir tabaco y cannabis se incrementa a medida que los adolescentes cuentan con más cantidad de dinero, sobre todo a partir de los $20 €\left(\chi^{2}=126,80 ; p<, 001\right)$. $\mathrm{Al}$ igual que ocurre en el caso de la hora de llegada a casa, esta pauta se mantiene independientemente del sexo y del grupo de edad, si bien es cierto que en los adolescentes de 12 y 13 años, el hecho de contar con más dinero parece incrementar en mayor medida la probabilidad de consumir tabaco que la de consumir ambas sustancias (Tabla 6).

Por último, en un intento de modelización, se realizó un análisis de regresión logística, con el objetivo de ex- 
Tabla 5. Consumo de tabaco y cánnabis y hora de llegada a casa de adolescentes escolarizados por sexo y grupo de edad. Galicia Occidental, 2016.

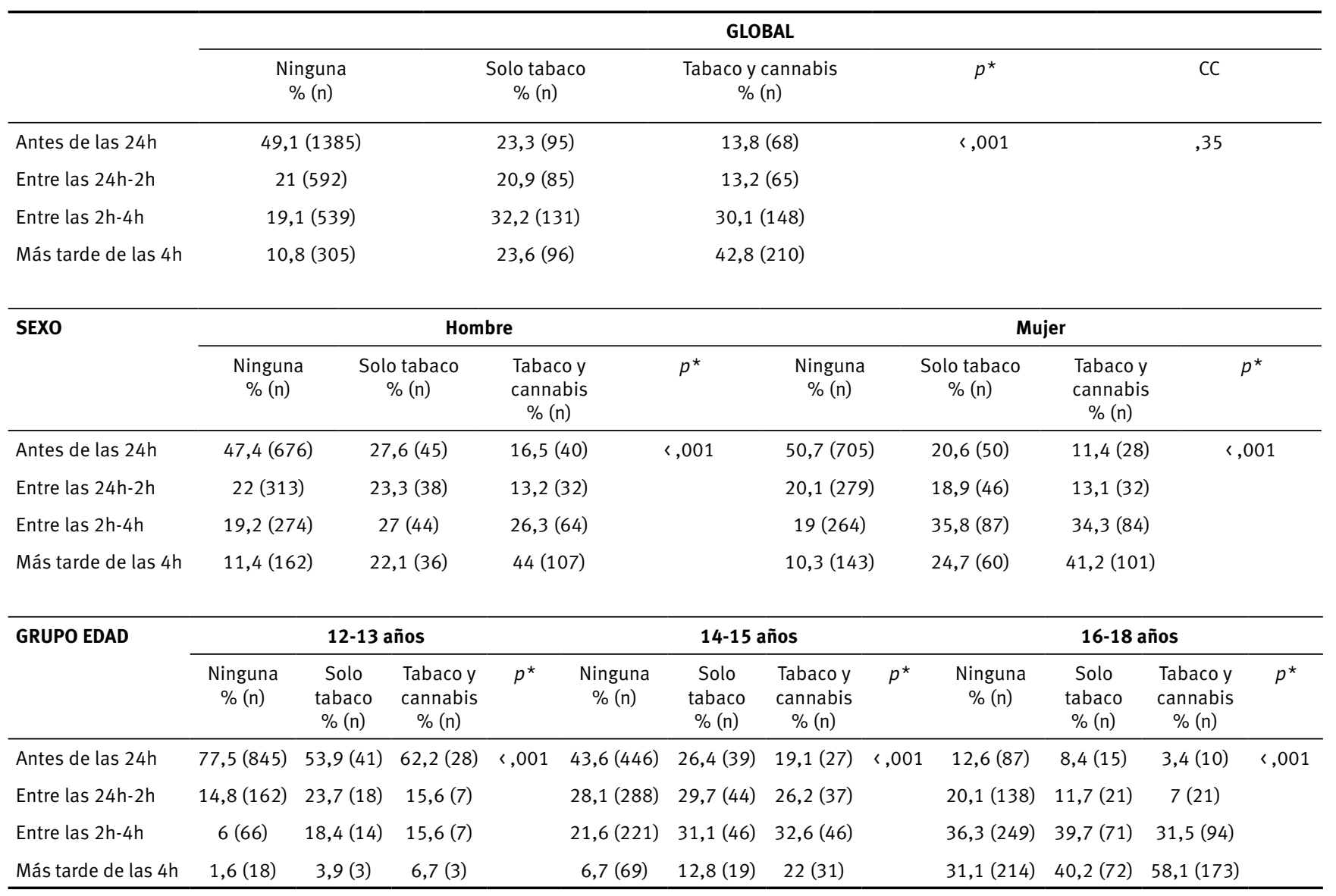

Nota. * Significación asociada al contraste Chi-cuadrado.

plorar en qué medida variables personales y familiares podrían explicar de manera conjunta el hecho de que los adolescentes consuman cannabis además de tabaco. Los resultados obtenidos, tanto para la muestra global, como de manera estratificada por sexo y grupo de edad, revelan una capacidad explicativa realmente pobre del modelo, con valores $\mathrm{R}^{2}$ de Nagelkerke nunca superiores al $10 \%$.

\section{Discusión}

El presente trabajo ha permitido constatar que la alarma social existente respecto al consumo de cannabis entre los adolescentes no resulta infundada, en la medida en que el consumo de dicha sustancia (y en particular el consumo de riesgo) se sitúa en niveles preocupantes. Ha quedado de manifiesto además que los adolescentes que "dan el salto" al consumo de cannabis tienen una mayor probabilidad de desarrollar un patrón de riesgo, caracterizado por un repertorio de consumo más perjudicial y una mayor probabilidad de implicarse en numerosas prácticas de riesgo en el uso de Internet.

Los resultados obtenidos revelan que los niveles de consumo encontrados en la muestra de adolescentes gallegos de entre 14 y 18 años se sitúan por debajo de los registrados en el ESTUDES 2014-2015 para el conjunto de España y ligeramente incluso que los obtenidos para Galicia (Plan Nacional sobre Drogas, 2016). A pesar de que cuando se incorpora la franja de edad de 12-13 años las cifras descienden notablemente, lo cierto es que el consumo de cannabis se mantiene en niveles preocupantes: en Galicia más de 10.000 adolescentes entre 12 y 18 habrían consumido cannabis en el último mes, por primera vez a una edad realmente muy temprana (14,8 años), coincidiendo con la primera borrachera. En el caso de los adolescentes de 12-13 años, a pesar de que el porcentaje de consumidores de cannabis en el último mes es de "tan solo" el 2,6\%, estaríamos hablando de más de 1000 niños/as, cifra nada despreciable, sobre todo si se tienen en cuenta las consecuencias que el consumo de cannabis a edades tan precoces puede acarrear tanto a nivel físico, como cognitivo, emocional y conductual (Brook, et al., 2008; Fergusson, Lynskey y Horwood, 1996; Filbey, McQueeny, DeWitt y Mishra, 2015). El análisis de los resultados en función del sexo y del grupo de edad pone de manifiesto algunas cuestiones que debieran ser tenidas en cuenta desde un punto de vista preventivo: a) conviene comenzar a prestar especial atención al colectivo de las mujeres, puesto 
Tabla 6. Consumo de tabaco y cánnabis y dinero disponible de los adolescentes escolarizados por sexo y grupo de edad. Galicia Occidental, 2016.

\begin{tabular}{|c|c|c|c|c|c|c|c|c|c|c|c|c|}
\hline & \multicolumn{12}{|c|}{ GLOBAL } \\
\hline & \multicolumn{3}{|c|}{$\begin{array}{l}\text { Ninguna } \\
\%(n)\end{array}$} & \multicolumn{2}{|c|}{$\begin{array}{c}\text { Solo tabaco } \\
\%(n)\end{array}$} & \multicolumn{2}{|c|}{$\begin{array}{c}\text { Tabaco y cannabis } \\
\%(n)\end{array}$} & \multicolumn{2}{|r|}{$p^{\star}$} & \multicolumn{3}{|c|}{$\mathrm{CC}$} \\
\hline Hasta $10 €$ & \multicolumn{3}{|c|}{$50,3(1436)$} & \multicolumn{2}{|c|}{$34,8(142)$} & \multicolumn{2}{|c|}{28,9 (142) } & \multirow{2}{*}{\multicolumn{2}{|c|}{$<, 001$}} & \multicolumn{3}{|c|}{,18 } \\
\hline Entre $11-20 €$ & \multicolumn{3}{|c|}{34,6 (988) } & \multicolumn{2}{|c|}{$43,6(178)$} & \multicolumn{2}{|c|}{42,1 (207) } & & & & & \\
\hline Entre $21-30 €$ & \multicolumn{3}{|c|}{$10,6(303)$} & \multicolumn{2}{|c|}{$16,2(66)$} & \multicolumn{2}{|c|}{$19,3(95)$} & & & & & \\
\hline Más de $30 €$ & \multicolumn{3}{|c|}{$4,4(126)$} & \multicolumn{2}{|c|}{$5,4(22)$} & \multicolumn{2}{|c|}{$9,8(48)$} & & & & & \\
\hline \multirow[t]{2}{*}{ SEXO } & \multicolumn{6}{|c|}{ Hombre } & \multicolumn{6}{|c|}{ Mujer } \\
\hline & $\begin{array}{l}\text { Ninguna } \\
\%(n)\end{array}$ & \multicolumn{2}{|c|}{$\begin{array}{l}\text { Solo tabaco } \\
\%(n)\end{array}$} & $\begin{array}{c}\text { Tabaco y } \\
\text { cannabis } \\
\%(n)\end{array}$ & & $p^{*}$ & $\begin{array}{l}\text { Ninguna } \\
\%(n)\end{array}$ & & $\begin{array}{l}\text { olo tabaco } \\
\text { \% (n) }\end{array}$ & $\begin{array}{c}\text { Tabaco y } \\
\text { cannabis } \\
\%(n)\end{array}$ & \multicolumn{2}{|r|}{$p^{\star}$} \\
\hline Hasta $10 €$ & $47,1(682)$ & \multicolumn{2}{|c|}{$32,3(53)$} & $26,7(65)$ & & $<, 001$ & $53,5(750)$ & & $36,6(89)$ & $31,3(77)$ & \multicolumn{2}{|c|}{$<, 001$} \\
\hline Entre $11-20 €$ & $35,2(509)$ & \multicolumn{2}{|c|}{$41,5(68)$} & \multicolumn{2}{|l|}{38,3 (93) } & & $34,1(478)$ & & 44,9 (109) & 45,5 (112) & & \\
\hline Entre $21-30 €$ & 12 (173) & 22 & (36) & $21,8(53)$ & & & 9,3 (130) & & $12,3(30)$ & $17,1(42)$ & & \\
\hline Más de $30 €$ & $5,7(83)$ & 4,3 & $3(7)$ & $13,2(32)$ & & & $3,1(43)$ & & $6,2(15)$ & $6,1(15)$ & & \\
\hline GRUPO EDAD & & $12-13 a$ & ños & & & $14-15$ & años & & & $16-18 a$ & años & \\
\hline & $\begin{array}{l}\text { Ninguna } \\
\%(n)\end{array}$ & $\begin{array}{c}\text { Solo } \\
\text { tabaco } \\
\%(n)\end{array}$ & $\begin{array}{c}\text { Tabaco y } \\
\text { cannabis } \\
\%(n)\end{array}$ & $p^{*}$ & $\begin{array}{l}\text { Ninguna } \\
\%(n)\end{array}$ & $\begin{array}{c}\text { Solo } \\
\text { tabaco } \\
\%(n)\end{array}$ & $\begin{array}{c}\text { Tabaco y } \\
\text { cannabis } \\
\%(n)\end{array}$ & $p^{*}$ & $\begin{array}{l}\text { Ninguna } \\
\%(n)\end{array}$ & $\begin{array}{c}\text { Solo } \\
\text { tabaco } \\
\%(n)\end{array}$ & $\begin{array}{c}\text { Tabaco y } \\
\text { cannabis } \\
\%(n)\end{array}$ & $p^{*}$ \\
\hline Hasta $10 €$ & $67,6(753)$ & $47,4(36)$ & $54,3(25)$ & $<, 001$ & $46(475)$ & $37,2(55)$ & 34,8 (49) &, 02 & 28,9 (199) & $27,9(50)$ & $22,1(66)$ & ,13 \\
\hline Entre $11-20 €$ & $23,8(265)$ & $31,6(24)$ & 28,3 (13) & & $38,2(394)$ & $43,9(65)$ & 39 (55) & & 46,9 (323) & $48,6(87)$ & 46 (137) & \\
\hline Entre $21-30 €$ & $5,9(66)$ & $15,8(12)$ & $10,9(5)$ & & $10,8(111)$ & 11,5 (17) & $16,3(23)$ & & $18(124)$ & $19,6(35)$ & $21,8(65)$ & \\
\hline Más de $30 €$ & $2,7(30)$ & $5,3(4)$ & $6,5(3)$ & & $5(52)$ & 7,4 (11) & 9,9 (14) & & $6,2(43)$ & $3,9(7)$ & $10,1(30)$ & \\
\hline
\end{tabular}

Nota. * Significación asociada al contraste Chi-cuadrado.

que en algunas sustancias (por ejemplo, el tabaco) las cifras de prevalencia ya son superiores entre las chicas, $y$ b) es necesario iniciar los esfuerzos a nivel preventivo a edades más tempranas, ya que se están registrando niveles preocupantes de consumo entre adolescentes de tan sólo 12 o 13 años.

En lo que respecta al consumo de riesgo, los resultados revelan que, a nivel global, un 3,8\% de los adolescentes de entre 12 y 18 años presentarían un consumo problemático de cannabis (positivo en el CAST), lo que significa que en 1 de cada 4 casos no se trata de un consumo ocasional o anecdótico. En la franja de edad de 14 a 18 años el porcentaje de consumidores de riesgo se sitúa en un 4,9\%, siendo el doble del registrado a nivel nacional (Plan Nacional sobre Drogas, 2016). El hecho de que los niveles de consumo de riesgo sean superiores a los obtenidos a nivel estatal, mientras que los niveles globales de consumo son inferiores, podría estar alertándonos respecto a un empeoramiento del patrón de consumo, compatible según algunos autores con un estancamiento del consumo de esta sustancia en términos absolutos (Isorna, 2017).

El análisis de la relación entre el consumo de tabaco y de cannabis, por su parte, revela que el consumo simultá- neo de ambas sustancias es mucho más frecuente de lo que se podría pensar. En la actualidad serían más los adolescentes que fuman tabaco y cannabis que solo tabaco. En el presente trabajo se ha constatado además que los adolescentes que realizan un consumo de ambas sustancias presentan no solo un mayor consumo intensivo de alcohol (binge drinking) y más borracheras, sino también un mayor consumo de riesgo, evaluado específicamente a través del AUDIT. También se han observado mayores tasas de consumo de otras sustancias, así como una mayor comorbilidad con otras conductas problema tales como o el Uso Problemático de Internet, el sexting, el ciberacoso o las apuestas onli$n e$. Todo ello coincide con los hallazgos de trabajos previos que han puesto en evidencia las implicaciones que tiene a distintos niveles el consumo simultáneo de ambas sustancias (Agrawal et al., 2009; Belánger, Akre, Kuntsche, Gmel y Suris, 2011; Hublet et al., 2015; Schauer, Rosenberry y Peters, 2017; Subramanian, McGlade y Yurgelun-Todd, 2016). De hecho, en el trabajo de Pinora et al. (2015), se ha constatado que el sistema endocannabinoide juega un papel importante en los efectos gratificantes y motivacionales de la nicotina. Por otra parte, la relación encontrada 
con el Uso Problemático de Internet y determinadas conductas potencialmente de riesgo en la Red fue advertida también en un reciente trabajo de Golpe et al. (2017) y hace pensar, tal y como propone la Teoría de la Conducta Problema (Jessor, 1991), que las diferentes conductas problema que con frecuencia emergen conjuntamente en la adolescencia podrían tener una base etiológica común. Los resultados obtenidos en función del sexo han servido para poner de manifiesto además que las posibles implicaciones del consumo simultáneo de tabaco y cannabis afectan tanto a chicas como a chicos, registrando incluso en algunos casos mayores porcentajes entre las chicas (como sucede, por ejemplo, en el consumo de riesgo de alcohol o en el ciberacoso). Atendiendo al grupo de edad, todo parece indicar que los adolescentes que consumen tabaco y cannabis son los que muestran una mayor probabilidad de consumir otro tipo de sustancias, independientemente de la franja de edad, así como de incurrir en conductas de riesgo en la Red, si bien en este último caso ello se hace especialmente visible a partir de los 14 años.

El presente trabajo constituía una buena oportunidad para explorar algunas de las variables que podrían explicar el consumo simultáneo de cannabis y tabaco. Ello resultaba de interés, habida cuenta de que la mayoría de los estudios suelen abordar los posibles factores de riesgo y de protección asociados bien a cada una de las sustancias de manera independiente, o bien al tabaco y al alcohol juntos, pero no al cannabis y al tabaco de forma simultánea (Álvarez et al., 2016). Los resultados obtenidos revelan que el consumo de ambas sustancias difícilmente puede ser explicado a partir de variables personales "clásicas", como la autoestima, la asertividad, la impulsividad o las habilidades sociales, relacionándose más bien con otras variables vinculadas al papel de los padres, tales como la hora de llegada a casa o el dinero disponible, hecho que ya fue advertido en los trabajos de Becoña et al. (2013), Llorens, Barrio, Sánchez, Suelves y ESTUDES Working Group (2011) o Varela et al. (2013). Como consecuencia, desde un punto de vista preventivo los resultados obtenidos recalcan la importancia de reforzar la prevención familiar en general y el papel de los padres, en particular. En este sentido, tal y como se sugiere en el trabajo de Burkhart (2011), resulta fundamental capacitarlos en el seguimiento ("monitoreo") de sus hijos (saber con quién y dónde están), así como en el establecimiento de una serie de normas y límites, habida cuenta la repercusión que ello tiene no solo sobre el consumo de sustancias psicoactivas, sino también sobre su socialización en general. Por último, desde un punto de vista institucional convendría seguir reforzando las políticas de control de tabaco y de cannabis, apostando también por un seguimiento continuado del problema y un modelo de prevención ambiental, que tenga en cuenta no solo las características personales del individuo, sino también el contexto cultural, social, físico y económico en el que éste se mueve (Burkhart, 2011).
En cuanto a las posibles limitaciones del presente trabajo, cabe referirse, en primer lugar, a la muestra utilizada. A pesar de haber contado con información de cerca de 4000 adolescentes, no cabe duda de que el hecho de haber optado por un muestreo no probabilístico para su selección y exclusivamente de las provincias de A Coruña y Pontevedra hace que los resultados deban ser interpretados con cierta cautela. Nuevos trabajos permitirán comprobar en qué medida los datos de prevalencia estimados pueden ser generalizables al resto de la comunidad gallega o incluso al conjunto del estado español. En segundo lugar, es posible que este estudio infraestime la prevalencia de consumidores de cannabis en adolescentes de 17 y 18 años en la medida en que los cursos académicos de $1^{\circ}$ y $2^{\circ}$ de Bachillerato no tienen carácter obligatorio y, por lo tanto, la muestra no incluye a los adolescentes de estas edades que han podido abandonar la enseñanza o seguir los estudios en los ciclos formativos de formación profesional. Asimismo, es importante referirse a la naturaleza transversal del trabajo, por lo que no es posible establecer relaciones de causalidad entre las variables objeto de estudio. Por otra parte, cabe destacar el limitado número de variables incluidas en el estudio, como posibles variables asociadas al consumo de cannabis, si bien éste no era el objetivo central del trabajo. Por último, conviene también hacer mención al hecho de que todas las variables han sido autoinformadas, por lo que es imposible conocer "a ciencia cierta" en qué medida los adolescentes pueden haber infraestimado o sobreestimado sus niveles de consumo. No obstante, como han señalado previamente diferentes expertos del ámbito de las conductas adictivas, las medidas de autoinforme han demostrado ser fiables e incluso mejores que otros métodos a la hora de evaluar los niveles de consumo de alcohol y otras drogas (Babor, Kranzler y Lauerman, 1989; Winters, Stinchfield, Henly y Schwartz, 1990).

Líneas de investigación futuras debieran plantearse la posiblidad de explorar e incorporar nuevas variables y centrar sus esfuerzos en elaboración de modelos explicativos parsimoniosos capaces de orientar la prevención de una manera eficaz. Asimismo, sería de gran interés desarrollar estudios longitudinales que permitieran esclarecer las relaciones de causa-efecto entre las variables.

\section{Reconocimientos}

Los autores quieren agradecer la financiación recibida a través de la Delegación del Gobierno para el Plan Nacional sobre Drogas (Ref. 2013/046) para la realización de este estudio.

\section{Conflicto de intereses}

Los autores declaran no tener ningún conflicto de intereses. 


\section{Referencias}

Agrawal, A., Lynskey, M. T., Madden, P. A. F., Pergadia, M. L., Bucholz, K. K. y Heath, A. C. (2009). Simultaneous cannabis and tobacco use and cannabis-related outcomes in young women. Drug and Alcohol Dependence, 101, 8-12. doi:10.1016/j.drugalcdep.2008.10.019.

Álvarez, A., Amatller, O., Esteban, A., Germán, I., Grifell, M., Isorna, M., ... Zabala, J. (2016). Proyecto ÉVICT Cannabis y tabaco. Informe 2015. Comité Nacional para la Prevención del Tabaquismo. Recuperado de http://www. evictproject.org/Informe_EVICT_2.pdf.

Ariza, C., García-Continente, X., Villalbí, J.R., Sánchez-Martínez, F., Pérez, A. y Nebot, M. (2014). Consumo de tabaco de los adolescentes en Barcelona y tendencias a lo largo de 20 años. Gaceta Sanitaria, 28, 25-33.

Arseneault, L., Cannon, M., Poulton, R., Murray, R., Caspi, A. y Moffitt, T. E. (2002). Cannabis use in adolescence and risk for adult psychosis: Longitudinal prospective study. The British Medical Journal, 325, 1212-1213. doi:10.1136/ bmj.325.7374.1212.

Asbridge, M., Hayden, J. A. y Cartwright, J. L. (2012). Acute cannabis consumption and motor vehicle collision risk: Systematic review of observational studies and meta-analysis. The British Medical Journal, 344, e536. doi:10.1136/bmj.e536.

Babor, T. F., Kranzler, H. R. y Lauerman, R. J. (1989). Early detection of harmful alcohol consumption: comparison of clinical, laboratory, and self-report screening procedures. Addictive Behaviors, 14, 139-157.

Barkus, E. (2008). Personality and cannabis use. Advances in Schizophrenia and Clinical Psychiatry, 3, 84-89.

Bechtold, J., Simpson, T., White, H. R. y Pardini, D. (2015). Chronic adolescent marijuana use as a risk factor for physical and mental health problems in young adult men. Psychology of Addictive Behaviors, 29, 552-563. doi:10.1037/ adb0000103.

Bélanger, R.E., Akre, C., Kuntsche, E., Gmel, G. y Suris, J.C. (2011). Adding tobacco to cannabis - its frequency and likely implications. Nicotine $\mathcal{E}$ Tobacco Research, 13, 746750. doi:10.1093/ntr/ntr043.

Becoña, E., Martínez, U., Calafat, A., Fernández-Hermida, J.R., Juan, M., Smnall, H., ... Gabrhelík, R. (2013). Parental permissiveness, control, and affect and drug use among adolescents. Psicothema, 25, 292-298.

Benotsch, E. G., Snipes, D. J., Martin, A. M. y Bull, S. S. (2013). Sexting, substance use, and sexual risk behavior in young adults. Journal of Adolescent Health, 52, 307-313. doi:10.1016/j.jadohealth.2012.06.011.

Brook, J. S., Stimmel, M. A., Zhang, C. y Brook, D. W. (2008). The association between earlier marijuana use and subsequent academic achievement and health problems: A longitudinal study. The American Journal of Addictions, 17, 155-160. doi:10.1080/10550490701860930.
Burkhart, G. (2011). Prevención ambiental de drogas en la Unión Europea. ¿Por qué es tan impopular este tipo de prevención? Adicciones, 23, 87-100.

Feingold, D., Weiser, M., Rehm, J. y Lev-Ran, S. (2016). The association beween cannabis use and anxiety disorders: Results from a population-based representative sample. European Neuropsychopharmacology, 26, 493-505. doi:10.1016/ j.euroneuro.2015.12.037.

Fergusson, D. M. y Boden, J. M. (2008). Cannabis use and later life outcomes. Addiction, 103, 969-976. doi:10.1111/ j.1360-0443.2008.02221.x.

Fergusson, D. M., Lynskey, M. T. y Horwood, L. J. (1996). The short-term consequences of early onset cannabis use. Journal of Abnormal Child Psychology, 24, 499-512. doi:10.1007/BF01441571.

Filbey, F. M., McQueeny, T., DeWitt, S. J. y Mishra, V. (2015). Preliminary findings demonstrating latent effects of early adolescent marijuana use onset on cortical architecture. Developmental Cognitive Neuroscience, 16, 16-22. doi:10.1016/ j.dcn.2015.10.001.

Font-Mayolas, S., Gras, M. E., Cebrián, N., Salamó, A., Planes, M. y Sullman, M. J. M. (2013). Types of polydrug use among Spanish adolescents. Addictive Behaviors, 38, 1605-1609.

George, T. y Vaccarino, F. (2015). The effects of cannabis use during adolescence. Ottawa, ON: Canadian Centre on Substance Abuse. Recuperado de http:// www.ccsa. ca/Resource Library/CCSA-Effects-of-Cannabis-Use-during-Adolescence-Report-2015-en.pdf.

Gerberich, S. G., Sidney, S., Braun, B. L., Tekawa, I. S., Tolan, K. K. y Quesenberry, C. P. (2003). Marijuana use and injury events resulting in hospitalization. Annals of Epidemiology, 13, 230-237. doi:10.1016/S10472797(02)00411-8.

Golpe, S., Barreiro, C., Isorna, M., Gómez, P., Varela, J. (2016, marzo). Antecedentes y consecuentes asociados a la edad de inicio de consumo de alcohol en adolescentes. Comunicación presentada en la XLIII Jornadas Nacionales de Socidrogalcohol, Alicante, España.

Golpe, S., Gómez, P., Braña, T., Varela, J. y Rial, A. (2017). Relación entre el consumo de alcohol y otras drogas y el uso problemático de Internet en adolescentes. Adicciones, 29, 268-277. doi:10.20882/adicciones.959.

González, M. P., Sáiz, P. A., Quirós, M. y López, J. L. (2000). Personalidad y uso abuso de cannabis. Adicciones, 12, 185-196.

Griffith-Lendering, M. F., Huijbregts, S. C., Huizink, A. C., Ormel, H., Verhulst, F. C., Volleberg, W. A. y Swaab, H. (2011). Social skills as precursors of cannabis use in young adolescents: a TRAILS study. Journal of Clinical Child E Adolescent Psychology, 40, 706-714. doi:10.1080/1 5374416.2011.597085.

Guerrero, M., Galván, G., Pinedo, J., Vásquez, F., Torres, F. y Torres, J. (2015). Prevalencia de vida de uso de can- 
nabis y rendimiento académico en adolescentes. Revista Cientifica Salud Uninorte, 31, 467-478. doi:10.14482/ sun.31.3.8046.

Guxens, M., Nebot, M., Ariza, C. y Ochoa, D. (2007). Factors associated with the onset of cannabis use: A systematic review of cohort studies. Gaceta Sanitaria, 21, 252-260. doi:10.1157/13106811.

Halbohn, C. (2016). The relationship between cyberbullying and health-risk behaviors among high school students. New York: Counselor Education Capstone. Recuperado de http:// digitalcommons.brockport.edu/cgi/ viewcontent. cgi? article $=1028 \&$ context=edc_capstone.

Harper, C., Dittus, P. y Ethier, K. (2016). Associations between marijuana use and 15 indicators of sexual risk and sexual health service use. Journal of Adolescent Health, 58, S109-S110. doi:1016/j.jadohealth.2015.10.230.

Hartman, R. L. y Huestis, M. A. (2013). Cannabis effects on driving skills. Clinical Chemistry, 59, 478-492. doi:10.1373/clinchem.2012.194381.

Henquet, C., Krabbendam, L., Spauwen, J., Kaplan, C., Lieb, R., Wittchen, H. U. y Van Os, J. (2005). Prospective cohort study of cannabis use, predisposition for psychosis, and psychotic symptoms in young people. The British Medical Journal, 330, 11-14. doi:10.1136/ bmj.38267.664086.63.

Hérnandez, T., Roldán, J., Jiménez, A., Mora, C., Escarpa, D. y Pérez, M. T. (2009). La edad de inicio en el consumo de drogas, un indicador de consumo problemático. Psychosocial Intervention, 18, 199-212.

Hindocha, C., Freeman, T.P., Ferris, J.A., Lynskey, M.T. y Winstock, A.R. (2016). No smoke without tobacco: A global overview of cannabis and tobacco routes of administration and their association with intention to quit. Frontiers in Psychiatry, 7, 104. doi:10.3389/fpsyt.2016.00104.

Hublet, A., Bendtsen, P., De Looze, M. E., Fotiou, A., Donnelly, P., Vilhjalmsson, R., ... Ter Bogt, T. F. (2015). Trends in the co-occurrence of tobacco and cannabis use in 15-year-olds from 2002 to 2010 in 28 countries of Europe and North America. European Journal of Public Health, 25, 73-75. doi:10.1093/eurpub/ckv032.

Hurd, Y. L., Michaelides, M., Miller, M. L. y Jutras-Aswad, D. (2014). Trajectory of adolescent cannabis use on addiction vulnerability. Neuropharmacology, 76, 416-424. doi:10.1016/j.neuropharm.2013.07.028.

Isorna, M. (2017). Cannabis: efectos, riesgos y beneficios terapéuticos. Vigo: Universidad de Vigo.

Jacobus, J. y Tapert, S. (2014). Effects of cannabis on the adolescent brain. Current Pharmaceutical Design, 20, 2186-2193. doi:10.2174/13816128113199990426.

Jessor, R. (1991). Risk behavior in adolescence: A psychosocial framework for understanding and action. Journal of Adolescent Health, 12, 597-605. doi:10.1016/1054139X(91)90007-K.
Kandel, D. B. (2003). Does marijuana use cause the use of other drugs? Journal of the American Medical Association, 289, 482-483.

Kokkevi, A., Gabhainn, S., Spyropoulou, M. y Risk Behaviour Focus Group of the HBSC (2006). Early initiation of cannabis use: A cross national European perspective. Journal of Adolescent Health, 39, 712-719. doi:10.1016/j. jadohealth.2006.05.009.

Legleye, S., Piontek, D. y Kraus, L. (2011). Psychometric properties of the Cannabis Abuse Screening Test (CAST) in a French sample of adolescents. Drug and Alcohol Dependence, 113, 229-235. doi:10.1016/j.drugalcdep.2010.08.011.

Llorens, N., Barrio, G., Sánchez, A., Suelves, J.M. y ESTUDES Working Group (2011). Effects of socialization and family factor on adolescent excessive drinking in Spain. Prevention Science, 12, 150-161. doi: 10.1007/s11121-0100195-0.

Malmberg, M., Overbeek, G., Monshouwer, K., Lammers, J., Vollebergh, W. A. y Engels, R. C. (2010). Substance use risk profiles and associations with early substance use in adolescence. Journal of Behavioral Medicine, 33, 474-485. doi:10.1007/s10865-010-9278-4.

Martín, J., Núñez, J. L., Navarro, J. G. y Grijalvo, F. (2007). The Rosenberg self-esteem scale: Translation and validation in university students. The Spanish Journal of Psychology, 10, 458-467.

Martínez, V., Fernández, J. R., Fernández, S., Carballo, J. L. y García, O. (2015). Adaptación y validación española de la Escala de Impulsividad de Barrat en adolescentes tempranos (BIS-11-A). International Journal of Clinical and Health Psychology, 15, 274-282.

Morales, E., Ariza, C., Nebot, M., Pérez, A. y Sáchez, F. (2008). Consumo de cannabis en los estudiantes de secundaria de Barcelona: inicio en el consumo, efectos experimentados y expectativas. Gaceta Sanitaria, 22, 321-329.

Moreno, M., Estévez, A. F., Zaldívar, F., García, J. M., Gutiérrez-Ferre, V. E., Esteban, L., ... Flores, P. (2012). Impulsivity differences in recreational cannabis users and binge drinkers in a university population. Drug Alcohol Dependence, 124, 355-362. doi:10.1016/j.drugalcdep.2012.02.011.

Morral, A. R., McCaffrey, D. F. y Paddock, S. M. (2002). Reassessing the marijuana gateway effect. Addiction, 97, 1493-1504. doi:1046/j.1360-0443.2002.00280.x.

Oliva, A., Antolín, L., Pertegal, M. A., Ríos, M., Parra, A., Hernando, A. y Reina, M. (2011). Instrumentos para la evaluación de la salud mental y el desarrollo positivo adolescente y los activos que lo promueven. Sevilla: Junta de Andalucía. Consejería de Salud.

Parada, M., Corral, M., Caamaño-Isorna, F., Mota, N., Crego, A., Rodríguez Holguín, S. y Cadaveira, F. (2011). De- 
finición del concepto de consumo intensivo de alcohol adolescente (binge drinking). Adicciones, 23, 53-63.

Pirona, A., Noor, A. y Burkhart, G. (2015, septiembre). Tobacco in cannabis joints: Why are we ignoring it? Póster presentado en Lisbon Addictions Conference 2015, Lisboa, Portugal.

Plan Nacional sobre Drogas. (2014). Encuesta sobre el uso de drogas en enseñanzas secundarias en España (ESTUDES) 2012-2013. Madrid, España: Delegación del Gobierno para el Plan Nacional sobre Drogas, Ministerio de Sanidad, Servicios Sociales e Igualdad.

Plan Nacional sobre Drogas. (2016). Encuesta sobre el uso de drogas en enseñanzas secundarias en España (ESTUDES) 2014-2015. Madrid, España: Delegación del Gobierno para el Plan Nacional sobre Drogas, Ministerio de Sanidad, Servicios Sociales e Igualdad.

Rial, A., Golpe, S., Araujo, M., Braña, T. y Varela, J. (2017). Validación del Alcohol Use Disorders Identification Test (AUDIT) en población adolescente española. Psicología Conductual, 25, 371-376.

Rial, A., Gómez, P., Isorna, M., Araujo, M. y Varela, J. (2015). EUPI-a: Escala de Uso Problemático de Internet en adolescentes. Desarrollo y validación psicométrica. Adicciones, 27, 47-63.

Rolle, I., Kennedy, S.M., Agaku, I. Everett, S., Bunnell, R., Caraballo, R.,... McAfee, T. (2015). Cigarette, cigar, and marijuana use among high school students - United States, 1997-2013. Morbidity and Mortality Weekly Report (MMWR), 64, 1136-1141

Rücker, J., Akré, C., Berchtold, A. y Suris, J. C. (2015). Problematic Internet use is associated with substance use in young adolescents. Acta Paediatrica, 104, 504-507. doi:10.1111/apa.12971.

Schauer, G.L., Rosenberry, Z.R. y Peters, E.N. (2017). Marijuana and tobacco co-administration in blunts, spliffs, and mulled cigarettes: A systematic literature review. Addictive Behaviors, 64, 200-211. doi:10.1016/j.addbeh.2016.09.001.

Silins, E., Horwood, L. J., Patton, G. C., Fergusson, D. M., Olsson, C. A., Hutchinson, D. M. y Spry, E. (2014). Young adult sequelae of adolescent cannabis use: An integrative analysis. The Lancet Psychiatry, 1, 286-293. doi:10.1016/S2215-0366(14)70307-4.

Subramanian, P., McGlade, E. y Yurgelun-Todd, D. (2016). Comorbid cannabis and tobacco use in adolescents and adults. Current Addiction Reports, 3, 182-188. doi:10.1007/ s40429-016-0101-3.

Swift, W., Coffey, C., Degenhart, L., Carlin, J. B., Romaniuk, H. y Patton, G. C. (2011). Cannabis and progression to other substance use in young adults: Findings from a 13 year prospective population-based study. Journal of Epidemiology Eீ Community Health, 66, 1-6. doi:10.1136/ jech.2010.129056.
Terzic, Z., Santric, M., Sbutega, I. y Vasic, V. (2013). The importance of family relations for cannabis users: The case of serbian adolescents. Iranian Journal of Public Health, 42, 249-260.

Thompson, R. G. y Auslander, W. F. (2007). Risk factors for alcohol and marijuana use among adolescents in foster care. Journal of Substance Abuse Treatment, 32, 61-69. doi:10.1016/j.jsat.2006.06.010.

Tullis, L.M., Dupont, R., Frost, K. y Gold, M.S. (2003). Marijuana and tobacco: A major connection? Journal of $\mathrm{Ad}$ dictive Diseases, 22, 51-62.

Van Rooij, A. J., Kuss, D. J., Grifftihs, M. D., Shorter, G. W., Schoenmakers, M. T. y Van de Mheen, D. (2014). The (co) occurrence of problematic video gaming, substance use, and psychosocial problems in adolescents. Journal of Behavioral Addictions, 3, 157-165. doi:10.1556/ JBA.3.2014.013.

Varela, J., Marsillas, S., Isorna, M. y Rial, A. (2013). El papel de las actitudes, las percepciones y el dinero disponible en el consumo de drogas en adolescentes. Salud y Drogas, 13, 67-78.

Vázquez, M. E., Muñoz, M. F., Fierro, A., Alfaro, M., Rodríguez, M. L. y Rodríguez, L. (2014). Consumo de sustancias adictivas en los adolescentes de 13 a 18 años y otras conductas de riesgo relacionadas. Pediatría de Atención Primaria, 16, 125-134.

Winters, K. C., Stinchfield, R. D., Henly, G. A. y Schwartz, R. H. (1990). Validity of adolescent self-report of alcohol and other drug involvement. International Journal of the Addictions, 25, 1379-1395. doi:10.3109/10826089009068469. 